\title{
Acute Meningitis in Mansoura Fever Hospital
}

\author{
Sameh M. Abdel Monem', Aya M. Al Amely ${ }^{1}$, Naglaa A. Khalifa ${ }^{2}$, \\ Omima T. Taha ${ }^{3 *}$, Ahmed L. Sharaf ${ }^{1}$
}

'Department of Tropical Medicine, Faculty of Medicine, Zagazig University, ${ }^{2}$ Department of Clinical Pathology, Faculty of Medicine, Zagazig University, '3Department of Obstetrics and Gynecology, Faculty of Medicine, Suez Canal University

\begin{abstract}
Background: Meningitis is a serious health problem that results in grave morbidity and mortality. It is caused by several organisms and present with various presenting pictures imparting some challenge. Aim: Highlighting the etiology and prognosis of acute meningitis in Mansoura Fever Hospital. Subjects and Methods: A prospective cross sectional observational study. The study included 350 cases admitted with suspected meningitis. Lumbar puncture: for collection of cerebrospinal fluid (CSF) samples. CSF analysis, microbiological examination of CSF, and blood culture and antibiotic sensitivity test were done. Brain magnetic resonant imaging (MRI) was performed before lumbar puncture in some patients. Detailed analysis of epidemiologic characteristics, clinical data and laboratory findings, the causative organisms, prognosis of all the patients with acute bacterial meningitis (ABM) were studied. Results: Meningitis occurred at all age groups. A significant proportion of meningitis patients $(60 \%)$ reported a positive history of antibiotic intake in the few days (3-5) before admission to the hospital. 79.1\% of ABM patients had a CSF leukocyte count in the range of $>100$ up to $1,000 \mathrm{cell} / \mathrm{mm} 3$ and $80 \%$ had CSF neutrophil percentage $>50 \%$. The pathogens were detected by direct Gram-stained smear in $86.4 \%$ of patients. Gram +ve bacteria was detected in 55/110 (50\%) of cases. Gram -ve bacteria detected in 40/110 (36.4\%) of cases. ABM carried a high mortality rate (22.7\%). ABM had $22.7 \%$ mortality rate. Conclusions: Meningitis is endemic in Egypt with a significant mortality rate. Strept. pneumonia was the most common offending organism.
\end{abstract}

Keywords: Acute bacterial meningitis; CSF markers; epidemiology; mortality.

\section{Introduction}

Meningitis is a drastic health concern, with multiple causative organisms that differ according to age and distribution. The rout of infection might be from the ears, nasopharynx, cranial injury, or congenital meningeal defect, or by blood stream spread ${ }^{(1)}$. Bacterial meningitis is associated with significant morbidity and mortality due to severe meningeal in- flammation ${ }^{(1)}$. More than one million people are affected each year globally and it is more common in developing countries as the meningitis belt in Africa (2). This belt stretches from Senegal to Ethiopia where the highest disease burden occurs. More than 400 million people are in danger of

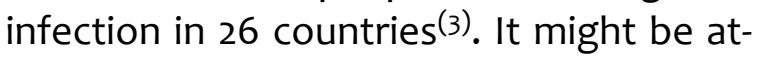
tributed to Escherichia coli, Neisseria meningitis, Streptococcus pneumonia, Haemophilus influenza, Streptococcus Group 
B, Staphylococcus aureus, and Listeria monocytogenes( ${ }^{(4)}$. On the contrary, viral meningitis, also called aseptic meningitis, is considered a self-limited disease with a benign course. Offending viruses include human herpes virus 6, herpes simplex (HSV), varicella zoster virus (VZV), cytomegalovirus (CMV), Epstein- Barr virus, JC virus and enterovirus(5). Non- infectious etiologies include malignant cells, drugs and blood following subarachnoid hemorrhage ${ }^{(6)}$. Although viral meningitis is more common, bacterial infection is more serious. Bacterial meningitis occurs at a rate of 40/100,000 in developing countries( 7 . The clinical picture may include definitive meningitis, encephalitis or meningism where the CSF is normal ${ }^{(8)}$. Bacterial meningitis is endemic in Egypt with Strept Pneumonia considered the leading offending organism $(42 \%)^{(9)}$. The disease is associated with significant long-term complications as epilepsy, hearing loss, hydrocephalus, and cognitive defects ${ }^{(10)}$. Significant morbidity and mortality occur in developing and developed countries ${ }^{(11)}$ with mortality rates reaching $5 \%$ and long-term morbidity of $15 \%{ }^{12)}$. Earlier studies conducted in Egypt reported high rates of morbidity and mortality $(9,13)$ highlighting the importance of updating the epidemiological aspects of such endemic disease in Egypt.

\section{Patients and Methods}

This cross-sectional study was conducted in Mansoura fever hospital from April 2019 to March 2020. Informed consent was obtained from all patients and the study protocol was approved by the ethical committee of Faculty of Medicine, Zagazig University. The study included 350 patients admitted to Mansoura fever hospital with suspected meningitis. Bacterial meningitis was confirmed when patients had fever, meningeal symptoms, altered mental status, and with an abnormal number of white blood cells in $\mathrm{CSF}^{(8)}$. Viral meningitis was considered in patients with symptoms suggestive of meningitis, absence of an identifiable cause (bacterial infection, fungal infection, autoimmune disease, injury, cancer, or drugs), and complete recovery with only conservative measures ${ }^{(14)}$. Patients were recruited according to inclusion and exclusion criteria. Inclusion criteria: a) any person with sudden onset of fever and one of the following signs: neck stiffness, altered consciousness, other meningeal signs or purpura fulminans, b) any child under 2 years of age with sudden onset of fever and one of the following signs: neck stiffness, or flaccid neck, bulging fontanel, convulsion, other meningeal signs or purpura fulminans. Exclusion criteria: Cases admitted with suspicious meningitis following head trauma or neurosurgical procedure, or cerebral abscess. All patients underwent history taking and clinical examination.

\section{Lumbar puncture}

CSF was collected from the subarachnoid space by a sterile spinal needle ( 25 or 27 $G)$ between the fourth and the fifth lumbar vertebrae. The CSF was collected in 2 sterile, screw-capped tubes. Two $\mathrm{ml}$ of CSF was collected in each tube. The first tube was labeled as No 1 (for direct Gram' stain, standard bacteriological culture methods and antibiotics sensitivity testing). The second tube was labeled as No 2 (for physical (color, aspect), chemical (glucose level, protein concentration) and cytological examination). A strict aseptic technique was used to collect the blood from patients. The top of the culture bottle was wiped using an ethanol swab, and $10-12 \mathrm{ml}$ of blood was taken.

\section{CSF analysis}

The specimen number one was centrifuged at 2000-3000 rpm for 20 minutes. 
The supernatant was aspirated with a sterile pipette, leaving approximately $0.5 \mathrm{ml}$ of fluid in the specimen tube (supernatant was reserved for biochemical studies), the sediment shaken to resuspend

- Physical characteristics of CSF: Color and aspect.

- Cytological examination: The presence of leukocytes was graded as: negative $(<10), 1+(10-50), 2+(51-290)$, and $3+$ (>291 cells/uL).

- Microbiological examination: Direct smears stained by Gram were done according to microbiological standards. Microscopical examination of Gram-stained smears was performed.

- Culture of CSF: Inoculation was done on blood, chocolate and Mac-Conkey's agar plates and incubated aerobically and anaerobically in presence of $5-10 \%$ $\mathrm{CO} 2$ at $37 \mathrm{C}$ for $72 \mathrm{~h}$. Identification of bacterial colony by colony morphology and biochemical reactions e.g.: Coagulase test, Catalase test, Optochin test and bile solubility test, Indole production test and Oxidase test.

Antibiotics sensitivity testing

Disk diffusion (Kibry-Bauer) method was used to determine the susceptibility of the recovered clinical isolates to antimicrobial agents. The diameters of inhibition zones were measured in $\mathrm{mm}$ using a ruler and classified as either susceptible, intermediate, or resistant to the agents that was tested.

\section{Brain MRI}

$M R I$ may be performed before the lumber puncture in patients with neurological deficit, seizure, Glasgow score $<11$.

Inpatient follow-up and outcome assessment

Patients were evaluated daily for symptom improvement or occurrence of new symptoms. Vital signs were assessed every four hours for the first 48 hours and based on the need thereafter. Daily follow-up with a neurosign chart that included the following variables: (Glasgow coma scale (GCS), seizure, headache, and nuchal rigidity) was done during the inpatient treatment. Patients were also assessed at discharge for gross neurologic deficits (visual problems, hearing deficit, and body weakness) and mini-mental state examination. Detailed analysis of epidemiologic characteristics, clinical data and laboratory findings, the causative organisms, prognosis of all the patients with $A B M$ were studied.

\section{Results}

We recruited 350 patients. One hundred and ten patients had confirmed diagnosis of acute bacterial meningitis (ABM) (Figure 1). $A B M$ occurred in males (61.8\%) more than females (38.2\%). It affected patients from urban (70.0\%) much more commonly than patients from rural (30\%) communities. Antecedent illnesses (i.e., any disease or condition which has given rise to another disease or condition in the morbid train of events leading to death) ${ }^{(13)}$ were found in $74.5 \%$ of patients with $A B M$. Pneumonia was the most common predisposing condition (33.6\%). $60 \%$ of meningitis patients reported a positive history of antibiotic intake in the few days before admission to the hospital (Table 1). The clinical presentation of ABM was characterized mainly by fever (90.9\%) and headache (88\%). Signs of meningeal irritation i.e., neck rigidity (90.9\%), Kernig sign (72.7), and Brudziniski sign (69\%) are significantly higher in patients with meningitis. Once neck rigidity, or headache, or both are present with fever meningitis is suspected (Table 2). $79.1 \%$ of ABM patients had a CSF leukocyte count in the range of $>100$ up to $1,000 \mathrm{cell} / \mathrm{mm} 3$ and $80 \%$ of $A B M$ patients had CSF neutrophil percent- 
age $>50 \% .85 .5 \%$ of $A B M$ patients had elevated CSF protein (> $8 \mathrm{omg} / \mathrm{dl}$ ). $81.8 \%$ of ABM patients had decreased CSF glucose (<50mg/dl). $90 \%$ of ABM patients had elevated CSF lactate $(>26 \mathrm{mg} / \mathrm{dl})$. Out of 110 CSF samples, 95 cases (86.4\%) pathogens were detected by direct Gram-stained sm- ear. Gram +ve bacteria were detected in 55/110 (50\%) of cases and Gram -ve bacteria in $40 / 110$ (36.4\%). Gram +ve cocci were the commonest (25/110) microorganism isolated in the present study. S. pneumonia was the most common isolated pathogen (24 isolates) (Table 3, and figure 2 ).

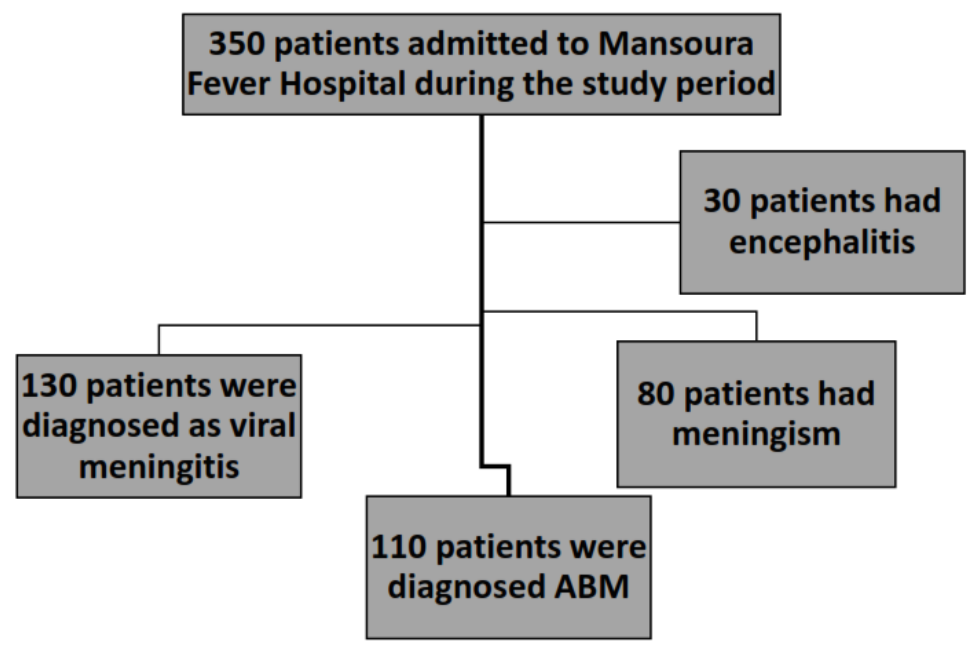

Table 1: Demographic data of $110 \mathrm{ABM}$ patients admitted to MFH

\begin{tabular}{|c|c|}
\hline Age (Yrs.) N (\%) & \\
$6-12$ month & $5(4.5 \%)$ \\
$>1-6$ & $10(9.1 \%)$ \\
$>6-18$ & $12(10.9 \%)$ \\
$>18-40$ & $25(22.7 \%)$ \\
$>40-60$ & $30(27.3 \%)$ \\
$>60$ & $28(25.5 \%)$ \\
\hline Sex N (\%) & \\
Male & $68(61.8 \%)$ \\
Female & $42(38.2 \%)$ \\
\hline Residence N (\%) & \\
Urban & $77(70.0 \%)$ \\
Rural & $33(30.0 \%)$ \\
\hline Antecedent illness N (\%) & \\
Otitis media & $16(14.5 \%)$ \\
Sinusitis & $21(19.1 \%)$ \\
Pneumonia & $37(33.6 \%)$ \\
Tonsillitis & $8(7.3 \%)$ \\
None & $28(25.5 \%)$ \\
\hline Spinal anesthesia N (\%) & $1(0.9 \%)$ \\
\hline Recent travel N (\%) & $6(5.5 \%)$ \\
\hline Antibiotic intake N (\%) & $66(60.0 \%)$ \\
\hline
\end{tabular}

Culture and sensitivity testing revealed ampicillin- sulbactam (53\%), cefotaxime (51.2\%), and ceftriaxone (47.3\%) as the effective antibiotics against gram + ve isolates. Vancomycin (70\%), piperacillin/ tazobactam (28\%), and levofloxacin (27.6\%) were revealed as sensitive antibiotics for those with gram -ve isolates. Blood cultures revealed the same organism as obtained by CSF culture in 73 (66.4\%) of cases. There was a different organism obtained in $14(12.7 \%)$ of cases. While negative culture was obtained in 23 (20.9\%) of cases. MRI was indicated before lumbar puncture if the case was suspected meningitis but with atypical presentation. MRI findings were as follows:

- Obstructive hydrocephalic changes at the level of aqueduct of Silvius with dilated both lateral ventricles and ballooned $3^{\text {rd }}$ ventricle with normal sized $4^{\text {th }}$ ventricle. 
- Ventriculitis which appeared as thick ependymal enhancement in ependymal lining of lateral ventricles on post contrast study as well as debris seen in both lateral ventricles forming fluid-fluid level. Theses debris displayed intermediate SI on both $\mathrm{T} 1$ and $\mathrm{T} 2 \mathrm{WI}$, restriction on DWI and no enhancement on post-contrast studies.

- Infarction along the territory of the middle cerebral artery (MCA) which appeared as wedge shaped areas cortical and subcortical in location fol- lowing MCA territory displaying low $\mathrm{SI}$ on $\mathrm{T} 1$ high $\mathrm{SI}$ on $\mathrm{T} 2$ and FLAIR images and restriction on DWI. It displayed mass effect in the form of effacement of the related cortical sulci and compression of ipsilateral lateral ventricle.

- Subdural empyema which appeared as well- defined crescent extra axial collection displaying low SI on T1 high $\mathrm{SI}$ on $\mathrm{T}_{2}$ and FLAIR, restriction on DWI, and thick marginal enhancement on post contrast study.

\begin{tabular}{|l|c|c|}
\hline \multicolumn{3}{|c|}{$\begin{array}{c}\text { Table 2: Clinical symptoms and signs } \\
\text { of the study patients }\end{array}$} \\
\hline Clinical picture & Frequency & Percentage \\
\hline Fever & 100 & $90.9 \%$ \\
\hline Headache & 97 & 88.2 \\
\hline Vomiting & 30 & $27.3 \%$ \\
\hline Photophobia & 18 & $16.4 \%$ \\
\hline Neck rigidity & 100 & $90.9 \%$ \\
\hline Irritability & 35 & $31.8 \%$ \\
\hline Skin rash & 10 & $9.1 \%$ \\
\hline Altered consciences & 18 & $16.4 \%$ \\
\hline Seizures & 16 & $14.5 \%$ \\
\hline Kernig sign & 80 & $72.7 \%$ \\
\hline Brudzinski sign & 76 & $69.1 \%$ \\
\hline Local nerve signs & 8 & $7.3 \%$ \\
\hline AF bulge (N=5) & 3 & $60 \%$ \\
\hline Abnormal cry (N=10) & 4 & $40 \%$ \\
\hline Weak suckling ( $\mathrm{N}=8$ ) & 6 & $75 \%$ \\
\hline \multicolumn{2}{|c|}{ Data is expressed as percentage and frequency. AF (anterior fontanel) }
\end{tabular}

$A B M$ carried a high mortality rate (22.7\%). One infant $6 \mathrm{~m}$, one female patient $40 \mathrm{yrs}$. old, one girl 16 yrs. old, and all other cases were above 55 years old. $21.8 \%$ of ABM patients developed permanent sequelae as hearing deficit, paralysis, cognitive slowness, and memory trouble (Table 4).

\section{Discussion}

ABM occurred at a rate of $31.43 \%$ while viral meningitis affected $37.14 \%$ of the studied population. The ratio of bacterial to aseptic meningitis cases vary between dif- ferent studies. In an earlier study conducted in Egypt, the majority of meningitis cases were possibly viral meningitis (42.86\%) while bacterial meningitis with no positive culture growth occurred at a rate of $17.13 \%$, and finally, bacterial meningitis cases that showed positive culture growth affected $5.08 \%(15)$. However, another study reported a lower rate of bacterial meningitis $(10.3 \%)^{(16)}$. This difference in the percentage of bacterial versus aseptic meningitis can be attributed to differences in the place and time of stud- 
ies done, so, in developing countries bacterial meningitis was the major constituent of meningitis cases, while in developed countries and especially after the implementation of anti-capsular vaccines, bacterial meningitis became less event in relation to viral meningitis. Cases of $A B M$ were distributed at all age group as agreed with previous results ${ }^{(15,17)}$.

Table 3: Frequency of pathogens causing ABM detected by CSF direct stained film

\begin{tabular}{|l|c|c|}
\hline Direct stained film & Frequency & $\%$ \\
\hline Gram +ve cocci & 25 & $22.7 \%$ \\
\hline Gram +ve diplococci & 20 & $18.2 \%$ \\
\hline Gram +ve bacilli & 5 & $4.5 \%$ \\
\hline Gram +ve chain & 5 & $4.5 \%$ \\
\hline Gram -ve diplococci & 17 & $15.5 \%$ \\
\hline Gram - ve coccobacilli & 13 & $11.8 \%$ \\
\hline Gram -ve bacilli & 10 & $9.1 \%$ \\
\hline No detected strains & 15 & $13.6 \%$ \\
\hline
\end{tabular}

Table 4: Prognosis of the cases of ABM

\begin{tabular}{|l|c|c|}
\hline Outcome & No. & $\%$ \\
\hline Cured & 61 & 55.5 \\
\hline${ }^{*}$ Died & 25 & 22.7 \\
\hline${ }^{* *}$ Sequalae & 24 & 21.8 \\
\hline
\end{tabular}

Data is expressed as percentage and frequency. *(One infant $6 \mathrm{~m}$, one female patient 40 years old, one girl 16 years old, and all other cases were above 55 years old). ** Most of these sequelae hearing deficit, paralysis, cognitive slowness, and memory trouble.

A previous study reported increased prevalence of meningitis among infants and children ${ }^{(18)}$. This may be due to environmental factors and the availability of children vaccines against common pathogens causing meningitis, as $H$. influenzae vaccine of children at the United States in $1990^{(19)}$. Also, the current study included a few numbers of infants and young children which affects the true reflection of disease distribution as most of them were referred to Mansoura University Child Hospital. About three- quarters of $A B M$ patients had antecedent illnesses with pneumonia the most common antecedent condition (33.6\%). This agreed with a previous study where lung infections preceded pneumococcal meningitis in $40 \%$ of patients $^{(20)}$. Clinical features give the clue to the early diagnosis of ABM by a physician; therefore, there are various clinical symptoms which can be quite helpful in the diagnosis process. The majority of patients presented with the classic clinical features of bacterial meningitis includes fever, headache, and stiff neck. This agreed with previously reported results(21,22). Other signs occurred at variable rates across studies $^{(20,23,24)}$. Some patients with meningitis have atypical presentation e.g. infants younger than one year and elderly patients ${ }^{(18)}$. These neurologic manifestations are related to the severity of the disease and the time interval before arrival to the hospital ${ }^{(16)}$. Accordingly, none of the symptoms and signs could accurately discriminate between patients with meningitis from those without it. Patients with suspected meningitis on clinical grounds should be referred for lumber puncture and CSF examination to settle the correct diagnosis ${ }^{(25)}$. Sixty- one cases of the recruited patients were referred for MRI before LP because of atypical presentation. Pathological MRI findings are frequently found in patients with acute bacterial meningitis such as leptomeningeal enhancement, hypo intensity of subcortical white matter underlying intense meningeal inflammation(26). Additionally sometimes the edema of the brain or widening of the extra-axial CSF spaces may be evident in meningitis which can be evident by non-contrast MRI sequences ${ }^{(27)}$. MRI is useful for diagnosis of patients with atypical presentation as well as its complications ${ }^{(28)}$. Bacterial meningitis is a lifethreatening disease that is associated with significant mortality and morbidity. This was supported by previous results ${ }^{(17)}$. 


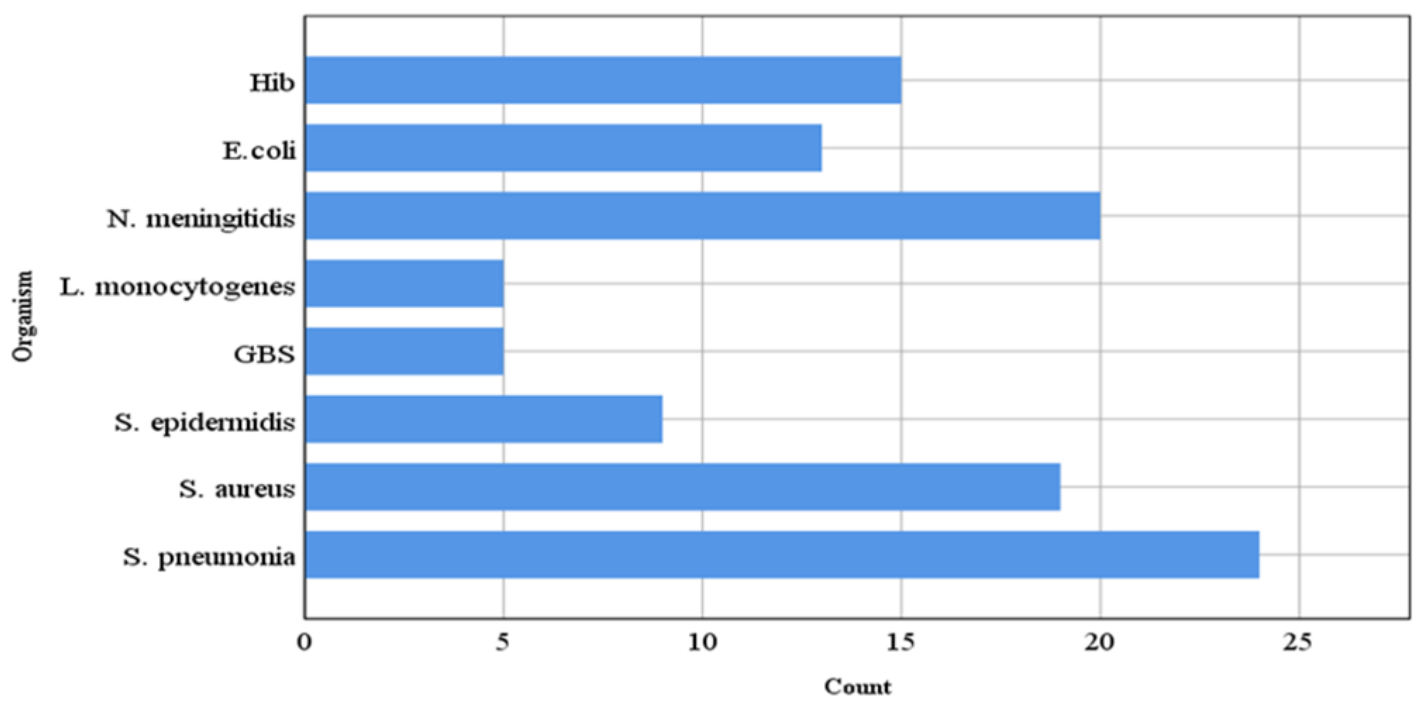

Figure 2: The frequency of bacteria causing $A B M$ by cultures

Without any treatment, the case-fatality rate can reach $70 \%$, and one in five survivors of bacterial meningitis may be left with permanent disability including hearing loss, neurologic disability, or limb loss ${ }^{(29)}$. The epidemiology of acute bacterial meningitis has changed markedly since the introduction of conjugate vaccines. However, the disease continues to cause a heavy burden even in developed countries, causing substantial morbidity and mortality ${ }^{(30)}$.

\section{Strength and limitations}

We recruited many patients. The diagnosis was done according to strict diagnostic criteria. MRI was done for patients with atypical presentation. However, the correlation between MRI findings and the clinical presentation was not done.

\section{Conclusion}

Acute bacterial meningitis affects a wide range of patients' age. Physicians should not neglect the atypical presentation of the disease to avoid drastic complications. $M R I$ is a useful tool for the diagnosis of atypical cases.

\section{Conflict of interest: None}

\section{References}

1- WHO. Meningococcal meningitis fact sheet. World Health Organization : http://wwwwhoint/

2- mediacentre/factsheets/fs141/en/, Geneva. 2017.1

3- Pfister W, Roos KL, 2003: Bacterial meningitis. In: Principles of Neurologic Infectious Diseases. Roos KL (ed.), McGraw-Hill.

4- Patel JC, Soeters HM, Diallo AO, et al. A Network Supporting CaseBased Meningitis Surveillance and Vaccine Evaluation in the Meningitis Belt of Africa, J Infect Dis, 220, Suppl_4, 1 Dec 2019, S148-S154.

5- Thigpen MC, Whitney CG, Messonnier NE, et al. Bacterial Meningitis in the United States, 19982007. N Engl J Med. 2011; 364 (21):2016-25.2

6- Nath A. Meningitis: bacterial, viral, and other. In: Goldman L, editor. Goldman-Cecil Medicine, 25th ed. Philadelphia, PA: Saunders; 2016. p. $2480-95 \cdot 3$

7- Jarvis JN, Meintjes G, Williams A, 
et al. Adult meningitis in a setting of high HIV and TB prevalence: findings from 4961 suspected cases. BMC Infect Dis. 2010; 10 (1):67.4

8- Brouwer M, Beek Dv. Epidemiology of community-acquired bacterial meningitis. Curr Opin Infect Dis 2018; 31 (1):78-84:

9- Jeffery KJ, Read SJ, Peto TE, et al. Diagnosis of viral infections of the central nervous system: clinical interpretation of PCR results. Lancet 1997; 349, 313-317. doi: 10.1016/S0140-6736(96)08107-X.

10- Afifi S, Wasfy MO, Azab MA, et al. Laboratory-based surveillance of patients with bacterial meningitis in Egypt (1998-2004). Eur J Clin Microbiol Infect Dis 2007; 26 (5):331-340.

11- Van de Beek D, de Gans J, Tunkel $A R$, et al. Community-acquired bacterial meningitis in adults. $\mathrm{N}$ Engl J Med 2006; 354:44-53.

12- Theodoridou MN, Vasilopoulou VA, Atsali EE, et al. Meningitis registry of hospitalized cases in children: epidemiological patterns of acute bacterial meningitis throughout a 32-year period. BMC Infect. Dis. 2007; 7:101-6.

13- Pelkonen T, Roine I, Monteiro L, et al, 2009: Risk factors for death and severe neurological sequelae in childhood bacterial meningitis in sub-Saharan Africa. Clin. Infect. Dis. 48:1107-10.

14- https://ec.europa.eu/eurostat/stati stics-explained/index.php/Glossary :Antecedent cause

15- Brivet FG, Ducuing S., Jacobs F. et al. Accuracy of clinical presentation for differentiating bacterial from viral meningitis in adults: a multivariate approach. Intensive Care Med 31, 1654-1660 (2005).

16- Ibrahim AA, Abdel Hakam SM, Ahmed AMH, et al. Updated Epidemiological Aspects and out- come of Meningitis Cases at Abbassia Fever Hospital during the Period 2006-2017. Afro-Egy J Infect Endemic Dis 2020;10(4):379-388

17- Abdelkader MM, Aboshanab KM, El-Ashry MA, et al. Prevalence of MDR pathogens of bacterial meningitis in Egypt and new synergistic antibiotic combinations. PLOS ONE. 2017; (2):1-17.

18- Amarilyo G, Alper A, Ben-Tov A, et al. Diagnostic Accuracy of Clinical Symptoms and Signs in Children With Meningitis. Pediatric Emergency Care. 2011; 27 (3):196-9.

19- Troendle M, Pettigrew A. A systematic review of cases of meningitis in the absence of cerebrospinal fluid pleocytosis on lumbar puncture. BMC Infect Dis. 2019; 19(1): 692-703.

20- Aneja S. Acute Bacterial Meningitis Early Diagnosis and Complications. Ind J Ped. 2015;82(4):303-5.

21- Dery $M$, Hasbun R. Changing epidemiology of bacterial meningitis. Curr Infect Dis Reports. 2007; $9(4): 301-7$.

22- Brouwer MC, Thwaites GE, Tunkel $A R$, et al. Dilemmas in the diagnosis of acute community-acquired bacterial meningitis. The Lancet. 2012;380 (9854): 1684-92.

23- Afifi S, Wasfy MO, Azab MA, et al. Laboratory-based surveillance of patients with bacterial meningitis in Egypt (1998-2004). Euro J Clin Microbiol \& Infect Dis. 2007; 26 (5):331-40.

24- Arda B, Sipahi OR, Atalay S, et al. Pooled Analysis of 2,408 Cases of Acute Adult Purulent Meningitis from Turkey. Med Principles and Practice. 2008;17(1):76-9.

25- Elmore JG, Horwitz RI, Quagliarello VJ. Acute meningitis with a negative gram's stain: Clinical and management out-comes in 171 episodes. Am J Med. 1996;100 (1):78-84. 
26- Heydarian F, Ashrafzadeh F, Rostazadeh A. Predicting factors and prevalence of meningitis in patients with first seizure and fever aged 6 to 18 months. Neurosciences. 2014; 19 (4): 297-300.

27- Brouwer MC, van de Beek D. Earlier Treatment and Improved Outcome in Adult Bacterial Meningitis Following Guideline Revision Promoting Prompt Lumbar Puncture. Clin Infect Dis. 2015; 61(4): 664-5.

28- Saberi A, Roudbary S-A, Ghayeghran $A$, et al. Diagnosis of Meningitis Caused by Pathogenic Microorganisms Using Magnetic Resonance Imaging: A Systematic Review. Basic and Clin Neurosci J. 2018;9(2):73-86.

29- Lummel N, Koch $M$, Klein $M$, et al. Spectrum and Prevalence of Pathological Intracranial Magnetic Resonance Imaging Findings in Acute Bacterial Meningitis. Clin Neuroradiol. 2014;26(2):159-67.

30- Tokimura $\mathrm{R}$, Iguchi $\mathrm{M}$, Ito $\mathrm{E}$, et al. The duration of antibiotic therapy in bacterial meningitis with pyogenic ventriculitis. Rinsho Shink eigaku. 2019;59(3):133-8.

31- Gudina EK, Tesfaye M, Wieser A, et al. Outcome of patients with acute bacterial meningitis in a teaching hospital in Ethiopia: A prospective study. PLOS ONE. 2018; 13(7): e0200067. 